\title{
Functionalization of gauzes with liposomes entrapping an anti-inflammatory drug: A strategy to improve wound healing
}

\author{
Helena Ferreira ${ }^{\mathrm{a}, \mathrm{b}}$, Teresa Matamá ${ }^{\mathrm{a}, \mathrm{c}}$, Raquel Silva ${ }^{\mathrm{a}}$, Carla Silva ${ }^{\mathrm{a}}$, Andreia C. Gomes ${ }^{\mathrm{c}}$, \\ Artur Cavaco-Paulo ${ }^{\mathrm{d}, *}$ \\ ${ }^{a}$ University of Minho, Department of Textile Engineering, Campus de Azurém, 4800-058 Guimarães, Portugal \\ ${ }^{\mathrm{b}}$ CICS, Health Sciences Research Sciences, Department of Pharmaceutical Sciences, Instituto Superior de Ciências da Saúde - Norte, Rua Central de Gandra, 1317, 4585-116 \\ Gandra-PRD, Portugal \\ ${ }^{\mathrm{C}}$ CBMA (Centre of Molecular and Environmental Biology), Department of Biology, University of Minho, Campus of Gualtar, $4710-057$ Braga, Portugal \\ ${ }^{\mathrm{d}}$ IBB-Institute for Biotechnology and Bioengineering, Centre of Biological Engineering, University of Minho, Campus de Gualtar, $4710-057$ Braga, Portugal
}

\section{A R T I C L E I N F O}

\section{Article history}

Available online 29 May 2013

\section{Keywords:}

Piroxicam

Wound healing

Inflammation

Liposomes

Nonwoven gauzes

\begin{abstract}
A B S T R A C T
From ancientness, suitable materials have been developed to cover the wounds in order to prevent infections and promote proper wound healing. In this study, the successful development of functionalized nonwoven gauzes with liposomes entrapping anti-inflammatory piroxicam is reported. Piroxicam is a non-steroidal anti-inflammatory drug (NSAID) that can suppress a persistent inflammatory response, leading to improved wound healing. The results demonstrated that the highest NSAID concentration released is achieved when gauzes were previously cationized with poly(diallyldimethylammonium chloride) (PDDA) and high concentration of phospholipid $(\approx 3000 \mu \mathrm{M})$ and multilamellar liposomes (MLVs) were used. MLVs were also the best vehicle considering their biocompatibility with skin human fibroblasts, where no toxicity was observed for neither of the tested conditions.

The developed functionalized gauzes can be, therefore, a good strategy to treat chronic wounds.
\end{abstract}

(c) 2013 Elsevier Ltd. All rights reserved.

\section{Introduction}

Wound healing is a complex process that includes inflammation, which is basically a defensive phenomenon yet often leading to serious pathological conditions if unbalanced. In fact, the inflammatory response is needed for the recruitment and activation of dermal cells to the wound site, which leads to the secretion of extracellular matrix, cell proliferation, angiogenesis, granulation tissue formation and eventual reepithelization of the wound [1]. Additionally, it has been well documented that an inflammatory response is needed to prevent infection, which if not eradicated impairs the healing process [1-3]. On the other hand, the state of chronic inflammation, that characterize chronic wounds, creates a proteolytic environment that is mediated by infiltration of inflammatory cells at the wound site, as well as prolonged overexpression of pro-inflammatory cytokines and chemokines that inhibit the normal progression of wound healing [2]. Therefore, non-steroidal anti-inflammatory drugs (NSAIDs), which are the principal pharmacological treatment for inflammatory diseases, could suppress the imbalances of chronic inflammation, leading to wound healing and restoration of the skin barrier

\footnotetext{
* Corresponding author. Tel.: +351 253510271; fax: +351 253510293.

E-mail address: artur@det.uminho.pt (A. Cavaco-Paulo).
}

function. This feature is very important, once that skin serves as a protective barrier against the environment and loss of cutaneous integrity, in the absence of adequate repair, can lead to major disability or even death [4].

Topical administration of NSAIDs can control severe side effects, as observed in gastrointestinal tract and kidney [5,6]. Besides this advantage over conventional oral and intravenous dosage forms, topical drug delivery offers many other benefits, such as first pass metabolism avoidance, pain minimization and patient compliance [7]. Consequently, there is a growing interest in the optimization of NSAID-carrier formulations to improve cutaneous delivery of these drugs. An example is liposomal formulations that have gained a considerable interest over the last decades. Additionally, advanced medical textiles is an area in expansion in the field of wound healing. Textile materials favour wound occlusion, exit of bacteria, exudates transport and drug dispensation with much reduced distress to the patient [8-10]. In addition, prolonged contact time of a drug with a bodily tissue, through the use of gauzes, for example, can significantly improve the clinical performance of many drugs. These improvements range from better treatment of local pathologies to improved drug bioavailability and controlled release, to enhanced patient compliance [11]. Thus, textiles' functionalization with drugs that counteract the progression of chronic wound inflammation is a promising approach to develop new textile-based wound dressings. 
In this context, the aim of this study was to entrap a NSAID, specifically piroxicam, into liposomes and then proceed to the attachment of those delivery systems to the nonwoven gauzes. Two types of liposomes were used, namely multilamellar liposomes (MLVs) and large unilamellar liposomes (LUVs), to investigate the best liposomal vehicle for the NSAID under study. MLVs present great variations in the vesicle size, size distribution and lamellarity, while LUVs are formed by a single bilayer of lipids, with well defined size $[12,13]$. Besides the liposomes, the influence of EPC concentration $(\approx 1500$ and $3000 \mu \mathrm{M})$ in liposomes properties and NSAID release it was also investigated. The production was followed by liposomes characterization which included the determination of size, polydispersity index (PDI), zeta-potential, morphology, drug entrapment efficiency and cytotoxicity. Liposomes were then attached to nonwoven gauzes to produce a functionalized biomaterial, with great potential for biomedical application. It was also explored if activation of the surface gauzes with a cationic product could lead to an enhancement of liposome attachment onto nonwoven gauzes. Finally, besides to evaluate the presence of liposomes in the gauzes surface, it was proceeded to the quantification of piroxicam released.

\section{Experimental}

\subsection{Materials and equipment}

The chemical reagents were purchased from Sigma-Aldrich and all were used as supplied. The nonwoven gauzes (composed by $67 \%$ of viscose and $33 \%$ of polyester, $3,1915 \pm 0,0077 \mathrm{~g} / \mathrm{m}^{2}$ ) were commercial available in a local pharmacy. The BJ5ta cell line (telomerase-immortalized human normal skin fibroblasts) was purchased from the European Collection of Cell Cultures (ECACC) and cultured according to American type culture collection (ATCC) recommendations.

The absorbance measurements were recorded at $25.0 \pm 0.1^{\circ} \mathrm{C}$ with a He $\lambda$ ios $\gamma$ ThermoSpectronic spectrophotometer (Unicam). A Spectraflash spectrophotometer (illuminant $D_{65}$ at $600 \mathrm{~nm}$ for Comassie Brilliant Blue G250 and $540 \mathrm{~nm}$ for Reactive Red 66), from Datacolor International, was used to measure the $K / S$ values.

The Malvern zetasizer NS (Malvern Instruments) equipment was used to determine the size distribution and the zeta-potential of the particles, using photon correlation spectroscopy (PCS) and electrophoretic laser doppler anemometry, respectively, at $25.0 \pm 0.1^{\circ} \mathrm{C}$.

The shape and morphology of liposomes, as well as their attachment into gauzes were observed using a scanning electronic microscope model NOVA Nano SEM 200 FEI.

The ultrasound equipment was composed of a probe type (20 kHz Sonics and Materials Vibracell CV 33) fitted with a $3 \mathrm{~mm}$ diameter titanium micro-tip. Power delivery was controlled as percentage amplitude (40\%) and $19 \mathrm{~mm}$ was the depth, measured from the base of the vessel, used. The reaction vessel was an open glass cell (diameter $19 \mathrm{~mm}$ and height $75 \mathrm{~mm}$ ), containing $16 \mathrm{~mL}$ of sample solution. The sonochemical reactor temperature was controlled via a thermo-stated water bath with a freezer exchanger placed within a thermo jacket cell.

The laboratory scale machine used to dye was an AHIBA Spectradye, from Datacolor International (Loorenstrasse 9, CH-8305 Dietlikon, Switzerland), with infrared temperature control.

\subsection{Liposomes preparation and drug incorporation}

Liposomes were prepared by the thin film hydration method [14]. A known amount of EPC was dissolved in chloroform and was mixed with a chloroform solution of piroxicam to obtain liposomes entrapping this drug. The organic solvent was then evaporated in a rotary evaporator and the lipid film formed was left at least $3 \mathrm{~h}$ to remove residual traces of the chloroform. The resulting dried lipid film was dispersed by addition of phosphate buffered saline solution (PBS; $0.01 \mathrm{M}, \mathrm{pH} 7.4$ ) and the resultant mixture was then vortexes above the phase transition temperature (room temperature) to yield MLVs. The sonication of MLVs suspension was carried out with a total treatment of $21 \mathrm{~min}$ monitored in $3 \mathrm{~min}$ increments, at $25 \pm 1{ }^{\circ} \mathrm{C}$. A pulsed duty cycle of $8 \mathrm{~s}$ on, $2 \mathrm{~s}$ off was used for all the experiments with indicated power delivery of $40 \%$. After LUVs preparation, the suspension was submitted to a centrifugation $(2500 \mathrm{~g}, 15 \mathrm{~min}$ ) to remove larger lipid aggregates and titanium particles released from the sonicator probe [15].

Liposomes were separated from non-entrapped drug by size exclusion chromatography using a Sephadex G-25 M column containing $0.15 \%$ kathan Gc as preservative (GE-Healthcare) and equilibrated with PBS buffer before use.

\subsection{Determination of size, PDI and zeta-potential}

Size distribution and zeta-potential values of EPC liposomes, with and without incorporated drug, were determined at $\mathrm{pH} 7.4$ (PBS), at $25.0 \pm 0.1{ }^{\circ} \mathrm{C}$. Lipid concentration was kept constant at approximately $500 \mu \mathrm{M}$.

\subsection{Quantification of NSAID entrapment efficiency in liposomes}

The entrapment efficiency of piroxicam into vesicular systems was evaluated by UV spectrophotometry, at $353 \mathrm{~nm}$. Entrapment efficiency refers to the ratio of quantity entrapped/adsorbed drug in relation to the total (theoretical) amount of drug used for liposomes production. Therefore, the entrapment efficiency of piroxicam was determined by:

Entrapment efficiency $(\%)=\frac{[\text { Piroxicam }]_{i}-[\text { Piroxicam }]_{f}}{[\text { Piroxixam }]_{f}}$

where $[\text { Piroxicam }]_{\mathrm{i}}$ and $[\text { Piroxicam }]_{\mathrm{f}}$ is the initial and final NSAID concentration, respectively, in supernatant after separation of the vesicular systems.

Measurements were recorded in triplicate and the results were expressed as mean value \pm standard deviation (SD).

\subsection{Determination of liposomes morphology}

The characterization of the overall structure and shape of liposomes was performed using STEM. For this analysis, the liposomes suspension was dropped in Copper grids with carbon film 400 meshes, $3 \mathrm{~mm}$ diameter.

\subsection{Chemical activation of gauzes}

The chemical activation of nonwoven gauzes comprised the cationization, as well as the determination of cationization efficiency on the gauzes surface.

The gauzes were cationized using the exhaust method already described [16]. The cationization was performed in sealed, stainless steel pots of $120 \mathrm{~cm}^{3}$ in capacity using a laboratory scale dyeing machine, previously referred in Section 2.1. The treatment was carried out using a material:liquor ratio of $1: 20,10 \%$ (of weight of the fabric) of poly(diallyldimethylammonium chloride) (PDDA) and $5 \mathrm{~g} \mathrm{~L}^{-1}$ of sodium hydroxide, over $60 \mathrm{~min}$ at $50{ }^{\circ} \mathrm{C}$. Afterwards, the gauzes were collected and washed thoroughly with deionised water. 
The cationization at the surface of nonwoven gauzes was analysed by staining of treated samples and controls (nonwoven gauzes without cationization treatment) with Comassie Brilliant Blue G250 or Reactive Red 66. The staining was executed in an AHIBA machine (referred in Section 2.1), using $1 \mathrm{~g} \mathrm{~L}^{-1}$ of dyes, at $60{ }^{\circ} \mathrm{C}$ for $60 \mathrm{~min}$. After staining, the samples were washed with deionised water until no more dye could be detected in the washing solution.

The colour measurements were determined using the spectrophotometer, previously referred in Section 2.1. The ratio between absorption coefficient $(K)$ and scattering coefficient $(S)$ is related to light reflection $(R)$ by applying Kubelka-Munk's law, at each wavelength, and it is proportional to dye concentration $[17,18]$ :

$\frac{K}{S}=\frac{1-R^{2}}{2 \times R}$

The colour strength was evaluated as $K / S$ at maximum absorption wavelength.

\subsection{Attachment of liposomes entrapping piroxicam onto gauzes}

The attachment of liposomes was achieved by incubating the different types of liposomes with the gauzes in a water shaking bath, at room temperature $\left(25^{\circ} \mathrm{C}\right)$, over 24 h under constant shacking $(50 \mathrm{rpm})$. After incubation, the samples were removed and washed with deionized water.

The presence of liposomes on gauzes samples was evaluated by SEM (the samples were coated with gold, in vacuum), and by using two dyes, namely Comassie Brilliant Blue G250 and Reactive Red 66 , present in two different concentrations, 0.25 and $1 \mathrm{~g} \mathrm{~L}^{-1}$. The colour measurements in the nonwoven gauzes were determined using the same conditions referred in Section 2.6. These tests were performed simultaneously with controls (nonwoven gauzes, cationized and non-cationized, without liposomes).

\subsection{Quantification of NSAID release from liposomes attached onto gauzes}

The release of piroxicam by the vesicular systems attached onto nonwoven gauzes $(\approx 0.07 \mathrm{~g})$ was assessed by incubation of the textile samples in deionised water ( $\mathrm{pH}$ 5.5), to mimic the skin pH (5.55.6) [19]. The volume of deionised water used was $3.5 \mathrm{~mL}$ and in the first $6 \mathrm{~h}$ was changed after every hour. After that, the medium was changed in each $24 \mathrm{~h}$. At determined time points, aliquots were taken, and the piroxicam release was monitored by absorbance measurements at wavelength of $356 \mathrm{~nm}$. The release studies were performed in triplicate and for a period of $72 \mathrm{~h}$.

\subsection{Cytotoxicity evaluation}

The cytotoxicity of LUVs and MLVs containing piroxicam was evaluated using normal human skin fibroblasts (BJ5ta). The BJ5ta cell line was maintained according to ATCC recommendations (4 parts Dulbecco's modified Eagle's medium (DMEM) containing $4 \mathrm{mM}$ L-glutamine, $4.5 \mathrm{~g} \mathrm{~L}^{-1}$ glucose, $1.5 \mathrm{~g} \mathrm{~L}^{-1}$ sodium bicarbonate and 1 part of Medium 199, supplemented with $10 \%(\mathrm{v} / \mathrm{v})$ of fetal bovine serum (FBS), 1\% (v/v) of Penicillin/Streptomycin solution and $10 \mu \mathrm{g} \mathrm{mL}^{-1}$ Hygromycin B). The cells were maintained at $37{ }^{\circ} \mathrm{C}$ in a humidified atmosphere of $5 \% \mathrm{CO}_{2}$. Culture medium was refreshed every 2 to 3 days. Cells were seeded at a density of $10 \times 10^{3}$ cells/100 $\mu \mathrm{L} /$ well on 96 -well tissue culture polystyrene (TCPS) plates (TPP, Switzerland), in the day before the experiments, and then exposed to different concentrations of liposomes entrapping or not piroxicam, as well as, of NSAID under study. At 24, 48 and $72 \mathrm{~h}$ of exposure, cell viability was determined using the alamarBlue $^{\circledR}$ assay (Invitrogen, EUA).
Table 1

Size (nm), PDI and zeta-potential (mV) values obtained for MLVs and LUVs of EPC $(\approx 1500$ and $3000 \mu \mathrm{M})$ incorporating piroxicam.

\begin{tabular}{|c|c|c|c|c|}
\hline \multicolumn{2}{|c|}{ Liposome } & \multirow[t]{2}{*}{ Size } & \multirow[t]{2}{*}{ PDI } & \multirow[t]{2}{*}{ Zeta-potential } \\
\hline Type & {$[\mathrm{EPC}](\mu \mathrm{M})$} & & & \\
\hline \multirow[t]{2}{*}{ MLVs } & 1500 & $1660 \pm 9.20$ & $0.99 \pm 0.01$ & $-1.31 \pm 0.05$ \\
\hline & 3000 & $1800 \pm 11.3$ & $1.00 \pm 0.00$ & $-2.06 \pm 0.08$ \\
\hline \multirow[t]{2}{*}{ LUVs } & 1500 & $66.76 \pm 2.01$ & $0.28 \pm 0.03$ & $-1.34 \pm 0.03$ \\
\hline & 3000 & $71.41 \pm 1.20$ & $0.31 \pm 0.09$ & $-1.90 \pm 0.02$ \\
\hline
\end{tabular}

Data are expressed as means with standard errors of the means. Two-way ANOVA followed by post hoc Bonferroni test (GraphPad Prism 5.0 for Windows) was employed with statistically significant differences when $P<0.05$.

\section{Results and discussion}

\subsection{Size distribution and zeta-potential of liposomes}

Table 1 shows the results of size and PDI obtained for liposomes incorporating piroxicam. As expected, the MLVs population presents a higher medium size and PDI, due to their heterogeneity.

The zeta-potential of the liposomes after separation of non-entrapped NSAID was also evaluated to obtain an indication of their surface potential. In Table 1 , it is also possible to verify that there was no significant difference on the zeta-potential values between LUVs and MLVs with piroxicam (the variations were smaller than $3 \mathrm{mV}$ ) and liposomes without drug $(\approx-1.01 \mathrm{mV})$. These results are in agreement with the presence of zwitterionic forms of this oxicam, at pH 7.4 [20]. Consequently, piroxicam has less influence over membrane surface potential.

\subsection{Liposomes morphology}

STEM microphotographs, present in Fig. 1, evidences that liposomes (MLVs and LUVs), with or without piroxicam, present a similarly spherical shape. The spherical shape observed, apart from other parameter, would offer the highest potential for controlled release and protection of incorporated drugs, as they provide minimum contact with the aqueous environment, as well as the longest diffusion pathways. Comparing particles with any other shape, spherical particles also require the smallest amount of surface-active agent for stabilization, because of their small specific surface area [21].

The results obtained with this technique were also in agreement with PCS results, once that the particle size distribution was very similar.

\subsection{Piroxicam entrapment efficiency}

The results obtained for the entrapment efficiency of piroxicam by LUVs and MLVs of EPC, present in different concentrations, are shown in Table 2. It is possible to conclude that the increase of phospholipid concentration promoted a small increment of NSAID entrapment, as expected, since piroxicam:EPC ratio was maintained. However, the results obtained for MLVs and LUVs, produced using the same EPC concentration, were significantly different. In fact, piroxicam may be located mainly between phospholipids in the lipid bilayer [20], due to its hydrophobicity, which also allows its solubilisation in chloroform and, consequently, its inclusion in the lipid film. Because LUVs present a higher curvature, this may hinder adequate packaging of piroxicam molecules. This data is also corroborated with literature, that refers multilamellar structures as being usually preferred to entrap hydrophobic 


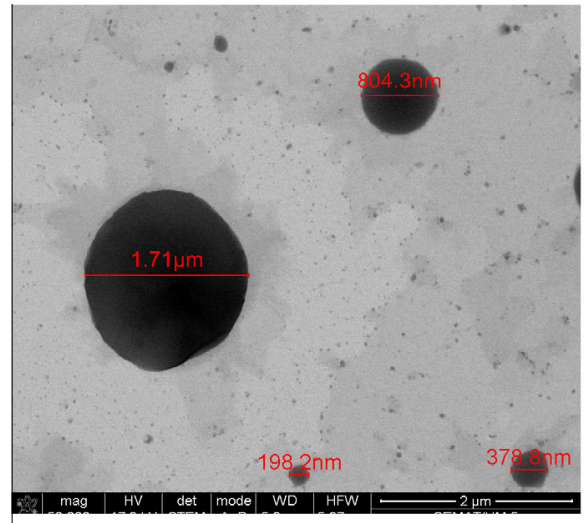

(a)

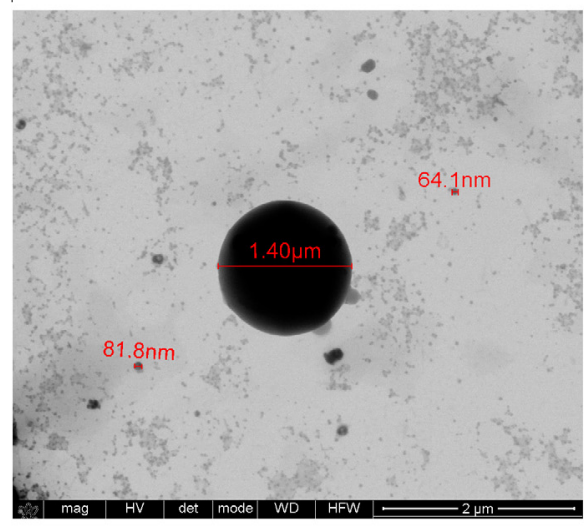

(c)

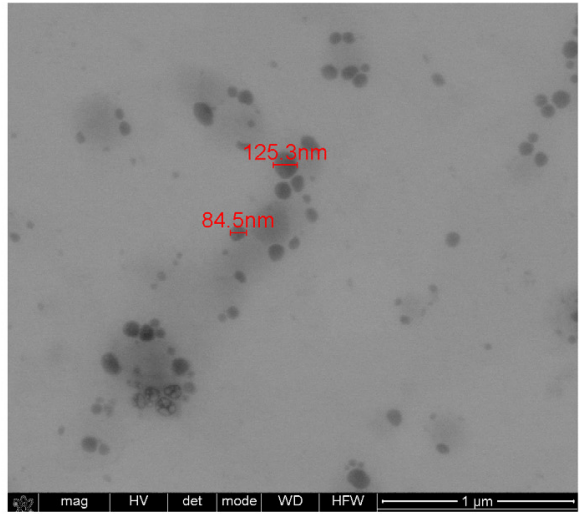

(b)

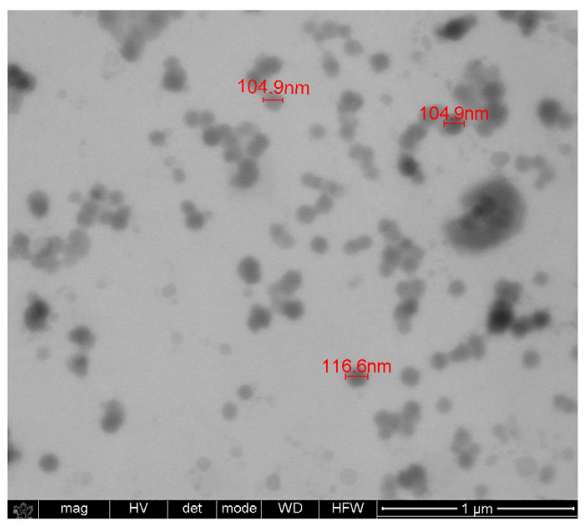

(d)

Fig. 1. STEM images of MLVs ( $\times 50000$ magnification) and LUVs ( $\times 100000$ magnification) without (a and b) or entrapping piroxicam (c and d).

Table 2

Entrapment efficiency (\%) of piroxicam into LUVs and MLVs of EPC present in different concentrations $(\approx 1500$ and $3000 \mu \mathrm{M})$.

\begin{tabular}{lll}
\hline Liposome & & Entrapment efficiency (\%) \\
\hline Type & {$[\mathrm{EPC}](\mu \mathrm{M})$} & \\
\hline MLVs & 1500 & $34.7 \pm 3.2$ \\
& 3000 & $36.6 \pm 4.0$ \\
LUVs & 1500 & $12.5 \pm 3.6$ \\
& 3000 & $17.2 \pm 2.7$ \\
\hline
\end{tabular}

molecules, while LUVs, having a large volume/surface, are more appropriate for the encapsulation of hydrophilic molecules [12,13].

\subsection{Attachment of liposomes entrapping piroxicam onto gauzes}

The potential of activating the nonwoven gauzes surface to attach the developed devices was explored. As the vesicules present negative surface charge, the activation of the gauzes surface, in order to obtain a positive charge, could be a good strategy to increase their attachment. It would promote a higher electrostatic interaction between the negative surface charge of the vesicular systems and the positive surface charge of the gauzes.

The attachment of the liposomes containing piroxicam, onto cationized and non cationized nonwoven gauzes, was evaluated using the dyes Comassie Brilliant Blue G250 and Reactive Red 66, as previously referred in Section 2.7. After staining, it was not possible to observe with naked eye a significant difference in colour between samples and controls, as obtained in a preliminary study performed with cotton fabric (data not shown). Consequently, it
Table 3

$K / S( \pm S D)$ values for the cationized and non-cationized nonwoven gauzes, with LUVs or $\operatorname{MLVs}(0, \approx 1500$ and $\approx 3000 \mu \mathrm{M}$ of EPC) entrapping piroxicam, dyed with Comassie brilliant blue $\mathrm{G} 250\left(1 \mathrm{~g} \mathrm{~L}^{-1}\right)$.

\begin{tabular}{lllll}
\hline \multicolumn{2}{l}{ Liposome } & & $K / S$ \\
\cline { 1 - 2 } Type & {$[\mathrm{EPC}](\mu \mathrm{M})$} & & Nonwoven non-cationized & Nonwoven cationized \\
\hline \multirow{2}{*}{ LUVs } & 0 & $0.83 \pm 0.05$ & $12.24 \pm 0.25$ \\
& 1500 & $0.64 \pm 0.02$ & $9.99 \pm 0.32$ \\
MLVs & 3000 & $0.61 \pm 0.04$ & $9.87 \pm 0.33$ \\
& 1500 & $0.72 \pm 0.03$ & $10.76 \pm 0.27$ \\
& 3000 & $0.67 \pm 0.02$ & $10.20 \pm 0.30$ \\
\hline
\end{tabular}

was necessary to measure the $K / S$ values in order to verify if the presence of liposomes led to a decrease of the colour strength, compared to controls, as verified in the preliminary study. Indeed, liposomes can form a lipid barrier at the gauze's surface, which impairs the binding of the dye to the fibre and, consequently, a decrease in $K / S$ values.

In Table 3, the $K / S$ values represent an example of the data obtained for the evaluation of liposomes entrapping piroxicam attachment using the dye Comassie. From this Table, it is possible to conclude that, as expected, the presence of liposomes onto gauzes contributes towards a reduction in $K / S$ values. Additionally, these results demonstrate a superior decrease of $K / S$ values for cationized samples, suggesting a higher attachment of the liposomes onto positively charged surfaces of the fibres. A higher decrease of $K / S$ values was also observed when the highest concentration of EPC was used, suggesting a superior liposome attachment in these experimental conditions. 
Finally, comparing the $K / S$ values of the controls of cationized and non-cationized gauzes (without liposomes), it is also possible to conclude that the cationization occurred efficiently, as the values are higher for the cationized samples. In fact, both dyes have more affinity for the cationized fibre, given the electrostatic interactions between their negative and the positive charge present on the cationized gauzes' surfaces.

The results obtained from the staining assays were corroborated by SEM analysis. Indeed, the morphological analysis of the fibres testifies the attachment of the liposomes containing piroxicam onto the nonwoven gauzes (Fig. 2).

\subsection{In vitro release profile of piroxicam from liposomes attached onto} gauzes

As shown in Fig. 3, the release of the anti-inflammatory drug occurs mainly in the initial hours. It is also possible to verify that when the highest concentration of phospholipid $(\approx 3000 \mu \mathrm{M})$ was used, the NSAID concentration in the aqueous phase increased. In fact, as previously referred, the greater is the number of MLVs or LUVs in suspension, the higher is the liposomes' attachment onto the substrate. Moreover, comparing the NSAID release profile from MLVs and LUVs, a higher concentration of piroxicam in the aqueous phase was obtained for the gauzes loaded with MLVs. This can be related with the size of this type of liposomes, once that being bigger favours retention onto the gauze's structure. Indeed, the porous nature of the textile influences the attachment of the particles once that, as previously referred in Section 3.4, with cotton fabric the differences in colour after samples dyeing were visible for the different liposome types. Nonwoven gauzes structure is less closed and compact than cotton fabrics, which can hold vesicular systems to a smaller extent, especially if they are smaller.

Comparing piroxicam concentrations released by the liposomes attached onto cationized and non-cationized gauzes (Fig. 3), it is possible to conclude that the activation of gauzes led to higher concentrations of the NSAID in the aqueous environment. Indeed, the enhancement of electrostatic interactions between liposomes and cationized gauzes promotes a higher yield of attachment and, consequently, of subsequent NSAID release.

\subsection{Cytotoxicity evaluation}

The biocompatibility of the two types of liposomes, without or with entrapped piroxicam, was assessed in normal human skin fibroblasts (BJ5ta cell line) upon 24, 48 and $72 \mathrm{~h}$ of contact. Before evaluating the biocompatibility of the different NSAID-liposome formulations, the cytotoxicity associated with the drug piroxicam was assessed in the same cell line. The results obtained showed that concentrations below $500 \mu \mathrm{M}$ of piroxicam did not significantly affect the viability of human skin fibroblasts, up to a maximum period of $72 \mathrm{~h}$ (cell viability above $80 \%$ ).
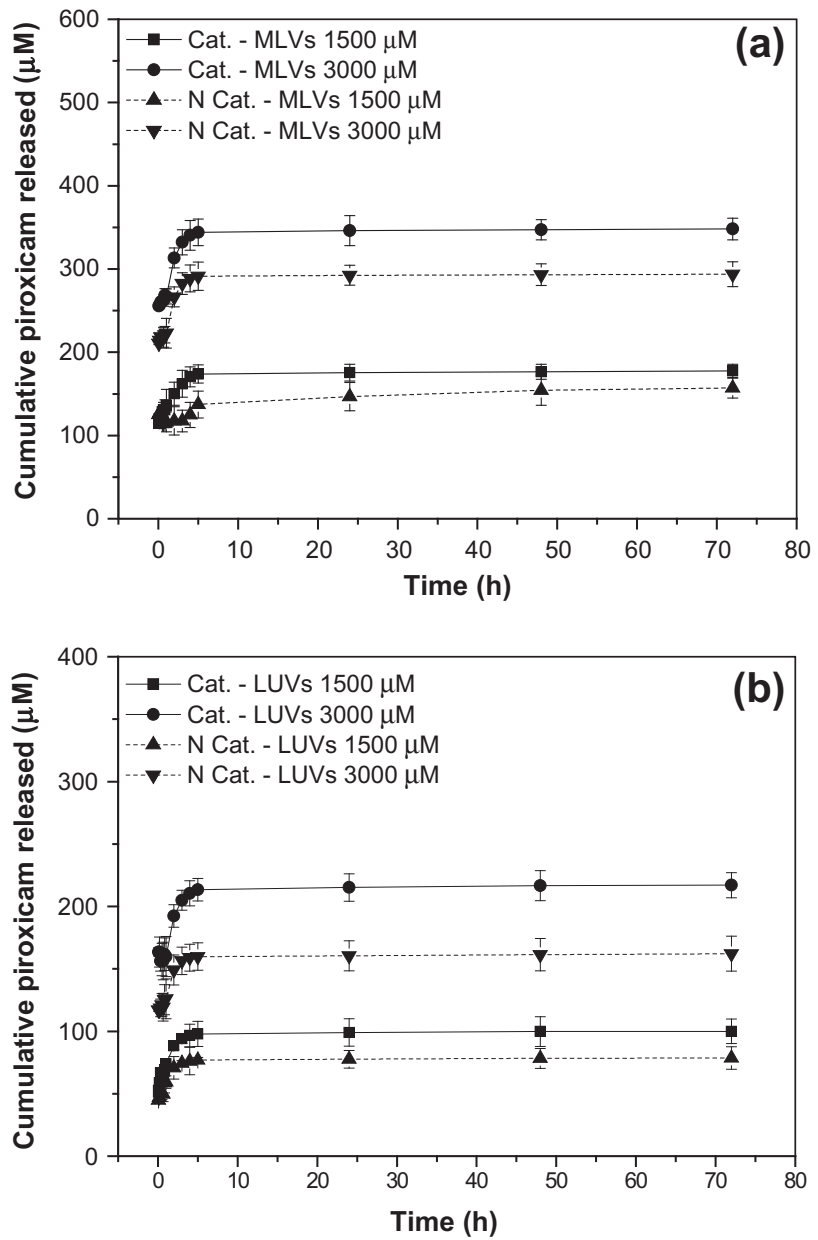

Fig. 3. In vitro release profile of piroxicam entrapped in MLVs (a) and LUVs (b) of EPC $(\approx 1500$ and $3000 \mu \mathrm{M})$, from cationized and non-cationized nonwoven gauzes.

The data, in Fig. 4, indicates that MLVs with or without entrapped piroxicam, at the tested concentrations and contact times, did not induce toxicity for the chosen mammalian cell model $(P>0.05)$. A similar result was obtained for the different concentrations of LUVs tested (Fig. 4). However, when this type of liposomes was enriched with piroxicam, statistically significant differences in relative toxicity were obtained for concentrations above $250 \mu \mathrm{M}$ of EPC when compared to the life control, for all exposure durations $(P<0.05$ for $500 \mu \mathrm{M}$ of EPC and $P<0.001$ for $750 \mu \mathrm{M}$ of EPC). Comparisons of different concentrations of LUVs containing piroxicam with the other tested samples were also performed. Statistically significant differences were obtained for the $500 \mu \mathrm{M}$ and $750 \mu \mathrm{M}$

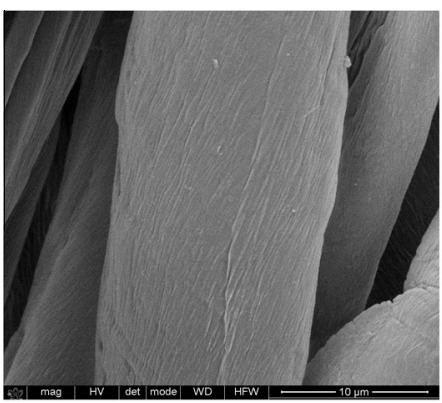

(a)

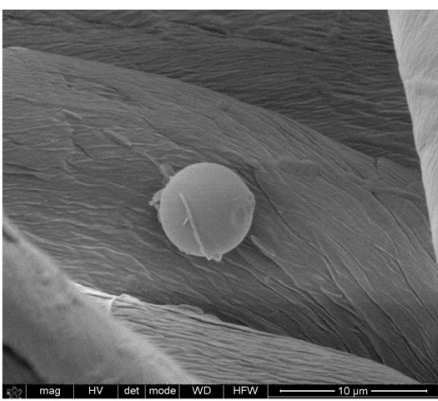

(b)

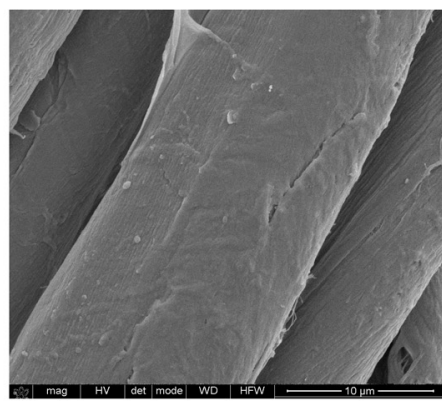

(c)

Fig. 2. SEM microphotographs ( $\times 10000$ magnification) of cationized nonwoven gauzes without liposomes (a) and with MLVs (b) or LUVs (c) entrapping piroxicam. 

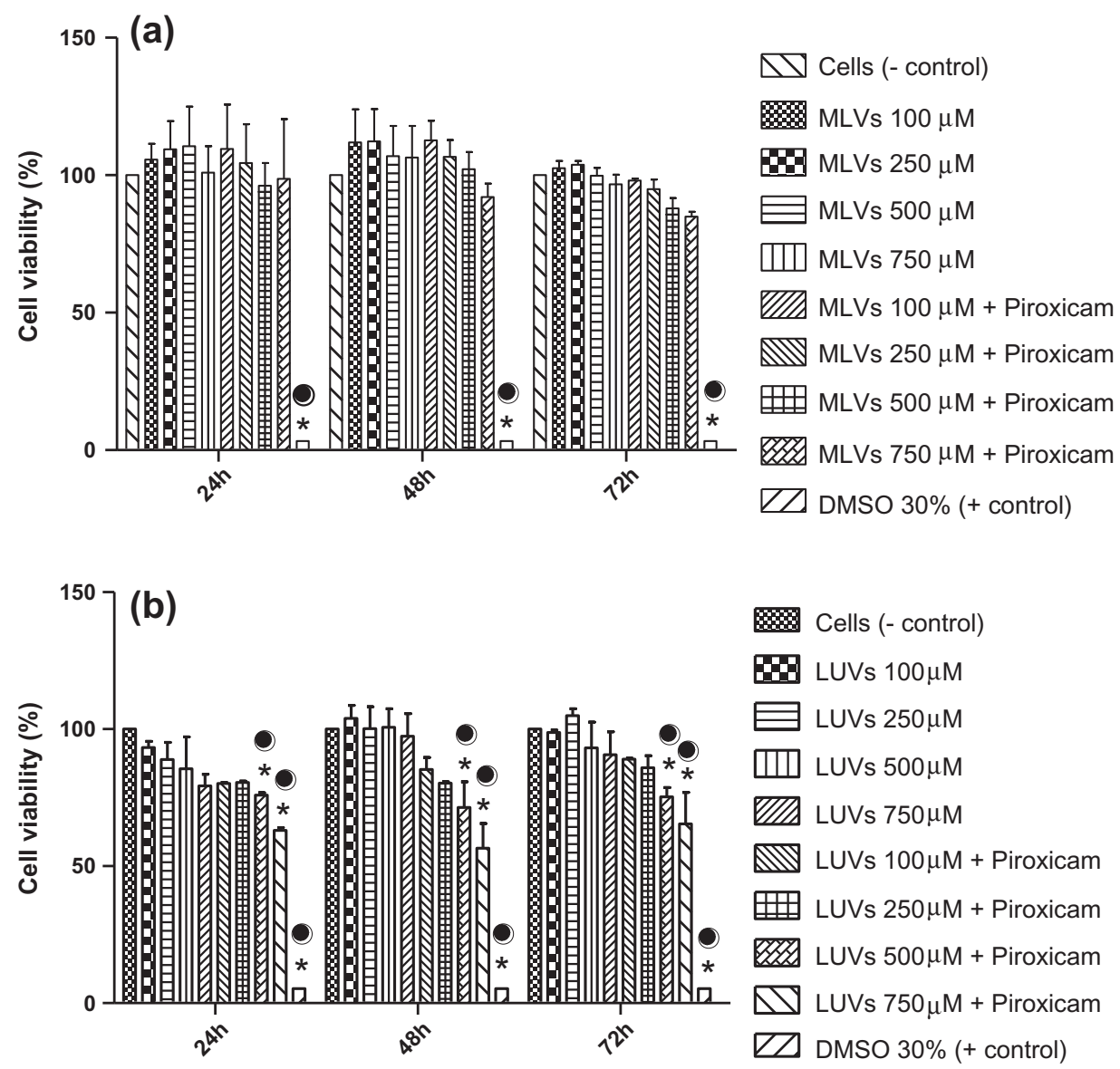

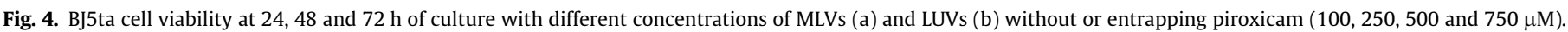

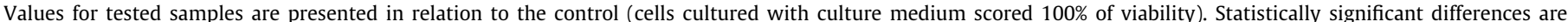

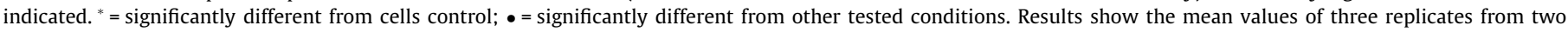
experiments carried out independently.

of EPC concentration, in the three experimental times considered. The toxicity achieved for these two samples can be related with the process of LUVs production. It is well known that ultrasonic irradiation of water produces free radicals [22], which contributes to lipid oxidation and to the modification of piroxicam chemical structure, given its antioxidant activity [23]. Nevertheless, the relationship between the NSAID concentration $(\approx 14 \mu \mathrm{M})$ in the $250 \mu \mathrm{M}$ of LUVs and the synovial fluid and plasma concentrations of piroxicam found in some studies after topical and oral administration [24], indicate that these liposomes can be suitable for the treatment of inflammatory conditions.

\section{Conclusions}

This work exploited the functionalization of nonwoven gauzes, with liposomes entrapping piroxicam, as a promising approach to develop new textile-based wound dressings.

The chemical activation of gauzes showed to be a good strategy to attach liposomes at the fibres surface, leading to higher NSAID concentration release into the aqueous environment. The same trend was observed by increasing EPC concentration, as well as by using MLVs, as drug carriers. In fact, MLVs showed to be the better vehicle even in terms of citotoxicity, as their cytocompatibility was verified for all conditions. In this sense, the functionalized gauzes developed are a promising tool to control topical chronic inflammatory conditions and boost wound healing processes.

\section{Acknowledgements}

Helena Ferreira, Teresa Matamá and Carla Silva thank POPH/FSE for co-financing and FCT for fellowships SFRH/BPD/38939/2007, SFRH/BPD/47555/2008, SFRH/BPD/46515/2008, respectively. This work was supported by FEDER through POFC - COMPETE and by national funds from FCT through the project PEst-C/BIA/UI4050/ 2011.

\section{References}

[1] J. Engelmayer, P. Blezinger, A. Varadhachary, J. Surgical Res. 149 (2008) 278286.

[2] I.T. Nizamutdinova, Y.M. Kim, J.I. Chung, S.C. Shin, Y.-K. Jeong, H.G. Seo, J.H. Lee, K.C. Chang, H.J. Kim, Food Chem. Toxicol. 47 (2009) 2806-2812.

[3] C.J. Beukelman, A.J.J. van den Berg, M.J. Hoekstra, R. Uhl, K. Reimer, S. Mueller, Burns 34 (2008) 845-855.

[4] C.R. Cardoso, S. Favoreto Jr, L.L. Oliveira, J.O. Vancim, G.B. Barban, D.B. Ferraz, J.S. Silva, Immunobiology 216 (2011) 409-415.

[5] J.B. Dahl, H. Kehlet, Br. J. Anaesthesia 66 (1991) 703-712.

[6] M.A. Álvarez-Soria, R. Largo, J. Santillana, O. Sanchez-Pernaute, E. Calvo, M. Hernandez, J. Egido, G. Herrero-Beaumont, Ann. Rheumatic Dis. 65 (2006) 9981005.

[7] M.M.A. Elsayed, O.Y. Abdallah, V.F. Naggar, N.M. Khalafallah, Int. J. Pharm. 332 (2007) 1-16.

[8] J.S. Boateng, K.H. Matthews, H.N.E. Stevens, G.M. Eccleston, J. Pharm. Sci. 97 (2008) 2892-2923.

[9] M. Miraftab, Q. Qiao, J.F. Kennedy, S.C. Anand, M.R. Groocock, Carbohydr. Polym. 53 (2003) 225-231.

[10] I.C. Gouveia, Polym. Adv. Technol. (2010). n/a-n/a.

[11] J.W. Lee, J.H. Park, J.R. Robinson, J. Pharm. Sci. 89 (2000) 850-866.

[12] D.D. Lasic, Liposomes - from Physics to Applications, Elsevier, New York, 1993. 
[13] R.R.C. New, Liposomes - A Practical Approach, Oxford University Press, New York, 1990.

[14] A.D. Bangham, R.W. Horne, J. Mol. Biol. 8 (1964) 660-668. IN662-IN610.

[15] C. Kirby, G. Gregoriadis, Biotechnology (1984) 979-984.

[16] M. Subramanian Senthil Kannan, M. Gobalakrishnam, S. Kumaravel, R. Nithyanadan, K.J. Rajashankar, V. Thilak, J. Textile Apparel Technol. Manage. 5 (2006) $1-22$.

[17] R.W. Harold, Textile Chem. Coloration 19 (1987) 23-31.

[18] R. Macdonald, Colour Physics for Industry Society of Dyers and Colourists, England, 1987.

[19] O. Braun-Falco, H. Korting, Hautarzt 37 (1986) 126-129.
[20] M. Lúcio, H. Ferreira, J.L.F.C. Lima, S. Reis, Med. Chem. 2 (2006) 447-456.

[21] H. Bunjes, Characterization of Solid Lipid Nanoparticles and Microparticle, in: C. Nastruzzi (Ed.), Lipospheres in Drug Targets and Delivery Approaches, Methods and Applications, CRC Press LLC, Boca Raton, Florida, 2005, pp. 41-66.

[22] R. Silva, C. Little, H. Ferreira, A. Cavaco-Paulo, Ultrason. Sonochem. 15 (2008) 1026-1032.

[23] M. Lúcio, H. Ferreira, J.L.F.C. Lima, S. Reis, Anal. Chim. Acta 597 (2007) 163170

[24] A.D.d. Jager, H. Ellis, H.K.L. Hundt, K.J. Swart, A.F. Hundt, J. Chromatogr. B 729 (1999) 183-189. 